$\stackrel{W}{=}$

Global journals Inc.

है

\title{
Results of Surgical Management of Highly Unstable Complex Distal Femur Fractures with Distal Femoral Locking Compression Plate Fixation: A Prospective Study of 58 Cases
}

By Dr. Rajesh Kumar Sharma \& Dr. Rajesh Goel

Rajasthan University of Health Sciences

Abstract-Background: The optimal treatment of complex distal femur fractures always remains challenging and controversial. The purpose of this prospective study was to evaluate the efficacy of distal femoral locking compression plate (DF-LCP) in terms of functional outcome, and union rate for highly unstable or complex distal femurfractures and to determine the influencing factors of an unfavorable outcome.

Methods: After obtaining approval from the institutional ethics committee, 58 patients with complex distal femur were managed by open reduction and internal fixation with DF-LCP through lateral approach and as per standard protocol. The follow-up results were analyzed clinically and radiologically, using the "Schatzker and Lambert criteria" at once in a month for the first three months, once in three months upto one year and once in six months after that up to two years.

Keywords: DF-LCP, lateral approach, schatzker and lambert criteria, secondary arthritis.

GJMR-H Classification: NLMC Code: WE 175

Strictly as per the compliance and regulations of:

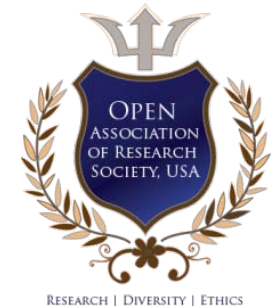

(c) 2020. Dr. Rajesh Kumar Sharma \& Dr. Rajesh Goel. This is a research/review paper, distributed under the terms of the Creative Commons Attribution-Noncommercial 3.0 Unported License http://creativecommons.org/licenses/by-nc/3.0/), permitting all noncommercial use, distribution, and reproduction in any medium, provided the original work is properly cited. 


\title{
Results of Surgical Management of Highly Unstable Complex Distal Femur Fractures with Distal Femoral Locking Compression Plate Fixation: A Prospective Study of 58 Cases
}

\author{
Dr. Rajesh Kumar Sharma ${ }^{\alpha} \&$ Dr. Rajesh Goel $^{\sigma}$
}

Abstract-Background: The optimal treatment of complex distal femur fractures always remains challenging and controversial. The purpose of this prospective study was to evaluate the efficacy of distal femoral locking compression plate (DF-LCP) in terms of functional outcome, and union rate for highly unstable or complex distal femurfractures and to determine the influencing factors of an unfavorable outcome.

Methods: After obtaining approval from the institutional ethics committee, 58 patients with complex distal femur were managed by open reduction and internal fixation with DF-LCP through lateral approach and as per standard protocol. The follow-up results were analyzed clinically and radiologically, using the "Schatzker and Lambert criteria" at once in a month for the first three months, once in three months upto one year and once in six months after that up to two years.

Results: In the present study, the average duration of the radiological union was 16 (range 12-24) weeks. The average range of motion of the knee joint was 105.5 degrees. Out of 58 patients, clinical results were excellent in $48.3 \%$, good in $19 \%$, fair in $22.4 \%$, and failure in $10.3 \%$ patients as per Schatzker and Lambert criteria. Knee stiffness (9 cases), secondary arthritis ( 5 cases), and non-union (4 cases) were the main complications observed in this study during two years of follow-ups.

Conclusion: The precontoured DF-LCP offers favorable clinical and radiological outcomes in the treatment of complex or highly unstable distal femur fractures with acceptable complication rates. It reduces impairment of periosteal blood supply due to limited plate-bone contact, provides angular stability, and rigid fixation of fragments regardless of bone quality, promotes early mobilization and rehabilitation even in osteoporotic, and severely comminuted fractures.

Keywords: DF-LCP, lateral approach, schatzker and lambert criteria, secondary arthritis.

Corresponding Author $\alpha$ : MS Orthopaedics \& Former Orthopaedic Resident (2015-18), Department of Orthopaedics, Govt. Medical College, Kota, Rajasthan, India.

e-mail: drrajeshsharma1986@gmail.com

Author o: Senior Professor \& Head of Department, Department of Orthopaedics, Govt. Medical College, Kota, Rajasthan, India, President of Rajasthan Orthopaedic Surgeons Association (ROSA 2020-21), Rajasthan, India. e-mail: doctorrajeshgoel@gmail.com

\section{INTRODUCTION}

( istal femur fractures are although uncommon, but usually challenging injuries for the treating Orthopedic surgeons. The overall incidence rate of these fractures is $<1 \%$, and $4-6 \%$ of all femoral fractures $[1,2]$. These fractures have a bimodal age group distribution. High energy injuries like road traffic accidents, sport's injuries, and falls from height are the prominent causes in younger patients, in contrast to elderly patients, where these fractures usually occur with low energy injuries like falls during walking and other household injuries [3, 4]. Distal femur fractures usually associated with compound injuries, severe comminution, and bone loss. On the other hand, proximity to the knee joint and unstable nature of the fracture makes it more prone to adverse functional outcomes. Inadequate management of such fractures have high incidences of infection, non-union, and malunion [5].

The management plan of these fractures depends on patient age, fracture grading, soft tissue injuries, and other associated injuries [6]. For treating Orthopedic surgeon, the ideal surgical goals are anatomical reduction of the fracture fragments, restoration of limb length, alignment and rotation, and rigid fixation that allows early mobilization and rehabilitation for the patient.

Before the 1970s, most of the distal femur fractures were treated conservatively with traction, casting, or combination of both. Due to prolonged bed rest, complications such as persistent angular deformity, bedsores, and loss of knee range of motion encountered in most of the patients [7, 8]. After the arrival of $\mathrm{AO}$ group, and upto the late 90s, many internal fixation devices used for the treatment of distal femoral fractures such as the dynamic condylar screw (DCS), or angled blade plate (ABP), condylar buttress plates, retrograde supracondylar inter-locking nails [9-11]. Although early mobilization was an advantage, rigid fixation in osteoporotic fractures and in severe metaphyseal comminutions were the main challenges. Other disadvantages were periosteal stripping and 
stress on implant lead to unfavorable outcomes, e.g. non-unions, and implant failures.

The distal femoral locking compression plate (DF-LCP) manufactured to overcome all these disadvantages. For highly comminuted and osteoporotic distal femur fractures, open reduction, and internal fixation (ORIF) with DF-LCP is gaining popularity nowadays. DF-LCP allows both locking and compression screw fixation of the femur shaft. The pullout strength of locking screws is significantly higher than that of typical screws, and it's arduous for one screw to pull out or fail unless all adjoining screws do the same. The favorable benefits of DF-LCP include stable angular fixation of fragments regardless of bone quality, reduced impairment of periosteal blood supply of the bone due to limited plate-bone contact, rigid fixation, early and active mobilization even in osteoporotic, and highly comminuted distal femur fractures [12-14].

The purpose of the present study was to evaluate the functional outcome, fracture union rate, and complications in highly unstable and osteoporotic fractures of distal femur treated with open reduction and internal fixation with distal femoral locking compression plates (DF-LCP) using Schatzker and Lambert criteria [13].

\section{Methods}

This study conducted during the years 2015 to 2018 in the Department of Orthopedics, Govt. Medical College, Kota (Rajasthan). Before the initiation of this study, approval of the institutional ethical committee was received. We designed a prospective study with a sample size of 58 patients with distal femur fractures, who met with inclusion criteria.

Inclusion Criteria: Skeletally matured patients with complex distal femur fractures (spiral, oblique, transverse, and butterfly fragment with intra-articular extension, and open fractures grade I \& II as per GustiloAnderson classification [15], osteoporotic fractures and had preparedness to take part in the study, were included.

Exclusion Criteria: Polytrauma patients, pathological fractures, periprosthetic fractures, existing deformity of the same limb, any active infection, open fractures grade III \& IV (as per Gustilo-Anderson classification [15], and fractures with neurovascular injuries excluded from the study.

All the mandatory preoperative routine investigations (blood and urine) done. To understand the morphology of fracture, an adequate radiological assessment, and 3-dimensional CT scan (especially in intra-articular femoral condyle fractures) carried out before the surgery. Lower tibial skeletal traction with proper weight was applied, in the situation of delayed surgery. We obtained the written informed consent from each patient before the procedures.

\section{a) Surgical Technique}

All surgeries performed by the same surgeons under spinal or combined spinal-epidural anesthesia. On the operating table, the patient placed in the supine position. Intravenous antibiotic (1 gm of Cephalosporin) injected 30 min before the surgery. We placed a pillow under the ipsilateral hip, and another one under the knee to obtain the flexed position of the knee. Depending on the length of the femur and proximal extension of fracture, a pneumatic tourniquet applied at the upper thigh in some patients. Routine preparations done such as scrubbing and draping of the injured limb.

The lateral standard approach used in all the patients. An incision parallel to the shaft of the femur, extending across the midpoint of the lateral femoral condyle, anterior to the lateral collateral ligament, across the knee, and gently curved anteriorly along the lateral border of the patella and up to the tibial tuberosity. The Vastus lateralis was elevated from the lateral inter muscular septum, and retracted anteriorly and medially, exposing the distal femur. The medial femoral condyle or coronal plane anatomy managed by adequate exposure of articular surface, and extension of the incision as per necessity.

The condyles were reduced and stabilized temporarily by $\mathrm{k}$ wires and fixed with $6.5 \mathrm{~mm}$ cannulated cancellous screws. The supracondylar part reduced, and the distal femoral locking compression plate placed. After putting a suction drain, the wound closure done in the standard manner.

\section{b) Post-operative follow-up}

Post-operatively intravenous antibiotics were given for five days, followed by oral antibiotics. Wound dressing checked on the second post-operative day. Routine post-operative $\mathrm{X}$-rays done before discharge. From 3rd day, continuous passive knee mobilization exercises twice daily were given to all the patients. Our purpose was to obtain at least 90 degrees of the knee flexion at the time of discharge. For the initial six postoperative weeks, all the patients directed to perform quadriceps, hamstring, and knee bending exercises properly. After six weeks, once the satisfactory clinical union ensured on examination, partial weight-bearing with leg knee brace support allowed. In our study, the clinical unionconsidered satisfactory, if the fracture site was pain-free, and two plane stability was present clinically at the fracture site. After 12 weeks, once enough radiological signs of fracture union detected in plane X-rays, full weight-bearing was allowed. It considered satisfactory radiological union, if plain radiographs showed at least three cortices of the bone or bone trabeculae crossing the fracture site. Although, the above mentioned protocol was delayed in case of delayed union.

Follow-ups were done regularly, once in a month for the first three months, once in three months 
upto one year and once in six months after that up to two years. At each follow-up, check $X$ rays taken, and all the information regarding postoperative complications, union time of fracture, partial weight-bearing time, full weight-bearing time of fracture recorded. Final assessment of all the patients was done at two years. For grading of the results, Schatzker and Lambert criteria [13] followed in this study.

\section{c) Statistical analysis}

The SPSS software version 16.0 and MS Excel 2013 used for statistical analysis. In the present study, qualitative variables demonstrated in proportion, and quantitative variables presented by the mean, and standard deviation.

\section{Results}

In this study, fifty-eight eligible patients operated during the study period from the years 2015 to
2018. Out of 58 patients, 40 were male, and 18 were female, with a mean age of 42.27 years (range 19-72). The mode of injury in 37 patients, was motor vehicle collision, in 19 patients, was fall from a height, and rest two patients presented with gunshot injury [Table 1]. These fractures were closed in 49 cases and compound in 9 cases ( 7 were Gustilo \& Anderson grade I, 2 were grade II).

The mean delay in operation was 7 (range 1-15) days. The mean duration of surgery was 80 (range 60110) minutes. The average perioperative blood loss was 250 (range 150-400) ml. The mean days of hospital stay were 12 (range 10-15) days. The various functional and radiological outcomes of our study, e.g. average time to weight-bearing, fracture union, ROM, and study results, are presented here in tabulated form [Table 2-4] and figures [Figure 1-4].

Table 1: Showing demographic variables of the study

\begin{tabular}{|c|c|c|}
\hline \multicolumn{2}{|c|}{ Demographic variables } & Features \\
\hline \multicolumn{2}{|c|}{ Study design } & Prospective study \\
\hline \multicolumn{2}{|c|}{ Study period } & 2015- 2018 \\
\hline \multicolumn{2}{|c|}{ Total number of the patients } & 58 \\
\hline \multirow{2}{*}{\multicolumn{2}{|c|}{$\begin{array}{c}\text { Male: Female } \\
\text { Mean age (range) in years }\end{array}$}} & $40: 18$ \\
\hline & & $42.27(19-72)$ \\
\hline \multirow{3}{*}{ Mode of injury } & Motor vehicle collision & 37 \\
\hline & Fall from height & 19 \\
\hline & Gunshot injury & 2 \\
\hline
\end{tabular}

Table 2: Showing various outcomes of the study

\begin{tabular}{|c|c|}
\hline Functional Outcome & Average duration (range) in weeks \\
\hline Partial weight bearing & $10(6-14)$ \\
\hline Full weight bearing & $16(12-24)$ \\
\hline Clinical union at fracture site & $10(6-14)$ \\
\hline Radiological union of fracture & $16(12-24)$ \\
\hline
\end{tabular}

Table 3: Showing knee range of motion in operated patients.

\begin{tabular}{|c|c|c|}
\hline $\begin{array}{c}\text { Post-op knee ROM* } \\
\text { (in degree) }\end{array}$ & Functional Outcome & $\begin{array}{c}\text { Number of patients } \\
\text { (n=58) }\end{array}$ \\
\hline 110 and more & Good to excellent & $28(48.3 \%)$ \\
\hline $91-109$ & Satisfactory & $13(22.4 \%)$ \\
\hline$<90$ & Unsatisfactory & $17(29.3 \%)$ \\
\hline
\end{tabular}

*ROM: Range of Motion

Table 4: Showing the functional outcome of the study.

\begin{tabular}{|c|c|c|}
\hline $\begin{array}{c}\text { Results } \\
\left.\text { (according to Schatzker \& Lambert criteria }{ }^{13}\right)\end{array}$ & $\begin{array}{c}\text { Number of patients } \\
(\mathrm{n}=\mathbf{5 8})\end{array}$ & Percentage of patients \\
\hline Excellent & 28 & $(48.3 \%)$ \\
\hline Good & 11 & $(19 \%)$ \\
\hline Fair & 13 & $(22.4 \%)$ \\
\hline Failure & 6 & $(10.3 \%)$ \\
\hline
\end{tabular}




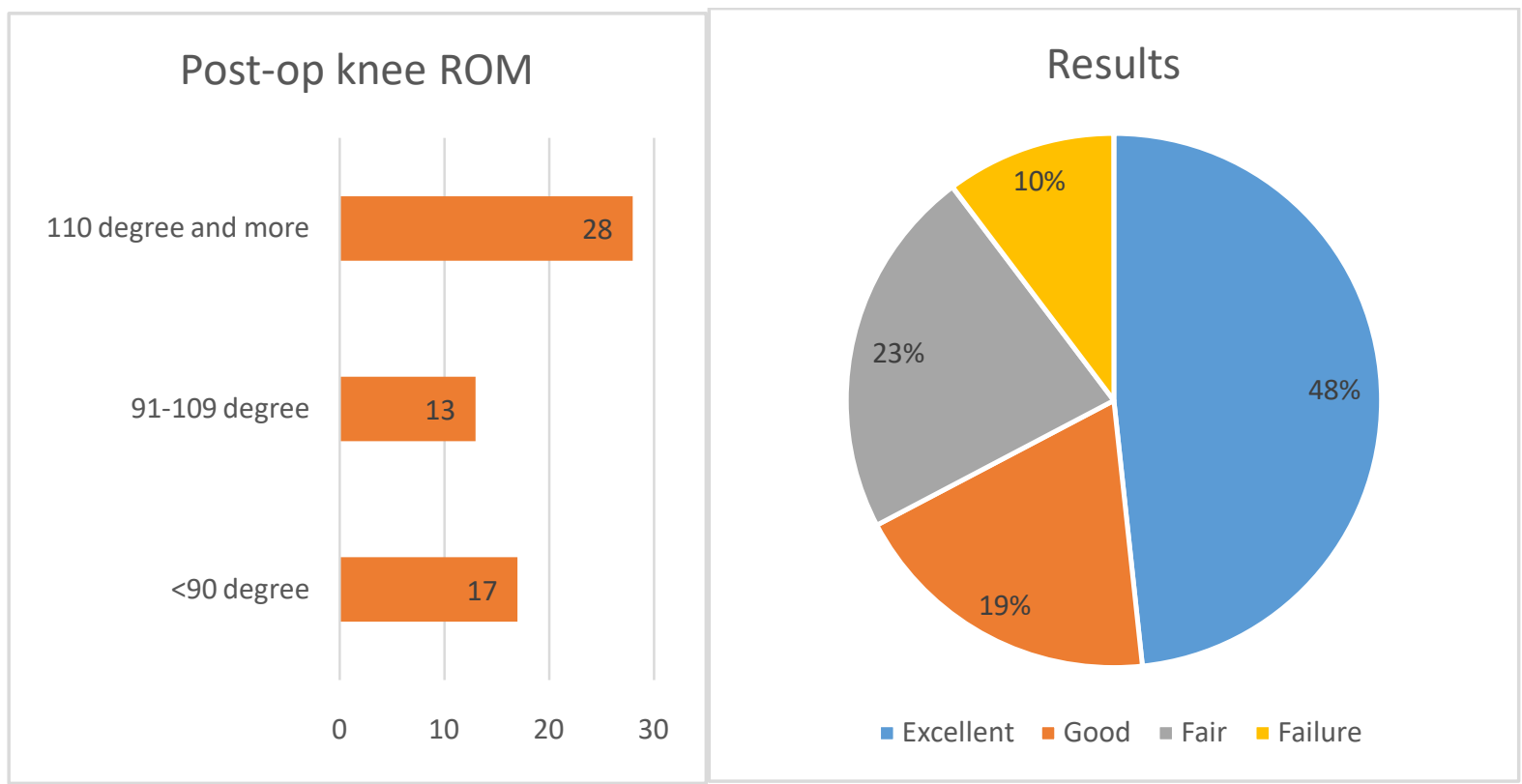

Figure 1: Showing knee 'Range of Motion' in post-operative patients and results of the study according to Schatzker \& Lambert's criteria

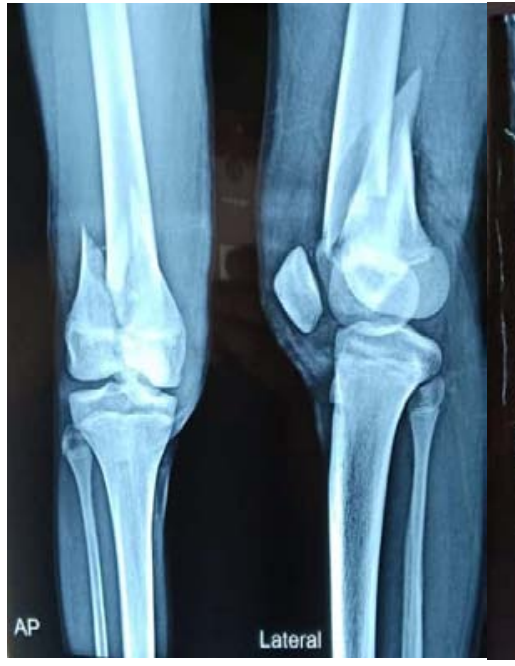

[Figure 2]

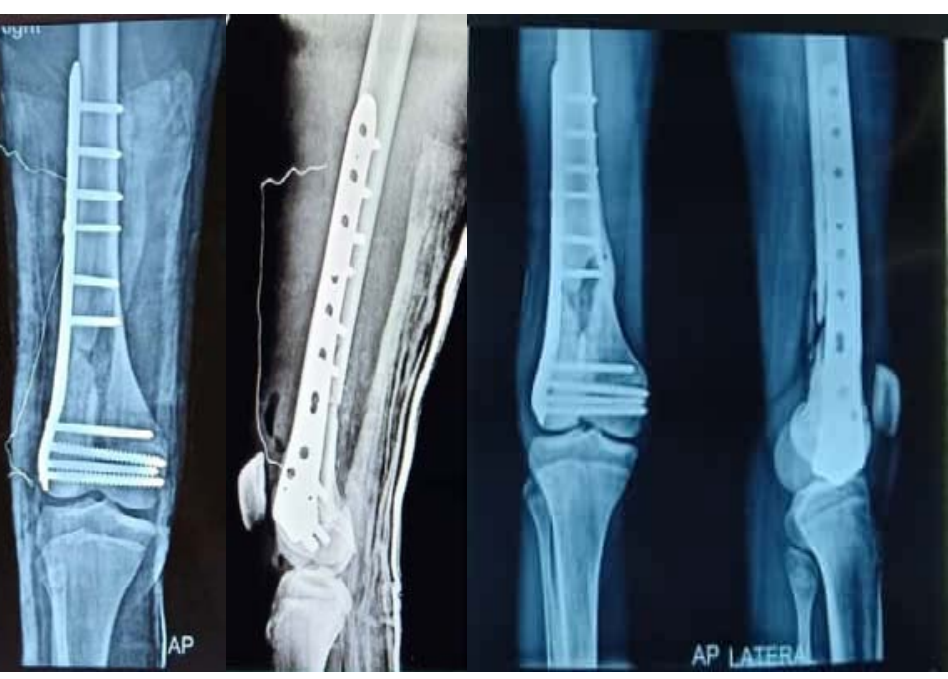

[Figure 3]

[Figure 4]

Figures 2-4: Evaluation of radiological (AP \& Lateral views) outcome of an unstable complex distal femur fracture, treated with DF-LCP fixation (pre-op, post-op, and at three months follow-up)

a) Complications of the study

We encountered some complications at the follow-ups of the patients. The most common complication was knee stiffness, observed in 9 (15.5\%) patients [Table 5].

Table 5: Showing the complications of the study

\begin{tabular}{|l|c|}
\hline \multicolumn{1}{|c|}{ Complications } & $\begin{array}{c}\text { Number of patients \& Percentage } \\
(\mathrm{n}=58)\end{array}$ \\
\hline Superficial surgical site infection & $3(5.2 \%)$ \\
\hline Deep infection & $2(3.4 \%)$ \\
\hline Delayed union & $2(3.4 \%)$ \\
\hline Knee stiffness & $9(15.5 \%)$ \\
\hline Limb lengths discrepancy or shortening $<2 \mathrm{~cm}$ & $3(5.2 \%)$ \\
\hline Implant failure & $3(5.2 \%)$ \\
\hline Non-union & $4(6.9 \%)$ \\
\hline Secondary arthritis & $5(8.6 \%)$ \\
\hline
\end{tabular}




\section{Discussion}

Surgical treatment methods for distal femur fractures are still controversial, and dependent on fracture type, and the surgeon's choice. Distal femoral locking compression plates (DF-LCP) have become the most commonly used procedure for internal fixation of distal $1 / 3$ rd femur fractures with or without intercondylar extension [16-18]. In DF-LCP, the sum of all screwbone interfaces gives the strength of fixation and makes it a 'single beam construct'. This plate has higher biological advantages than a standard plate [19]. It doesn't hamper the blood supply to the bone and maintains the cortical thickness of the bone, unlike standard plate.

In our study, the average age of patients, was 42.27 (range 19-72) years. This finding is almost comparable with the study of Siliski et al. [20] in which they reported the mean age of their study population as 42.2 years. Males were affected more commonly than females. The in present study, out of 58 patients, 40 patients (69\%) were male and 18 patients were female. It explained in such a way that working male adults were more involved in outdoor activities in a country like India and got such fractures more commonly. Similarly, 63\% were male patients in the study of Yeap et al. [21].

In the present study, clinical union assessed at ten weeks (mean), while radiological union was observed at 16 weeks in most of the patients. Although, the delayed union was also observed in two cases (3.4\%), in which union occurred at the end of 24 weeks of follow-up. Our study results are comparable with the results of previous studies of Rajaiah et al. [22], and Kim et al. [23]. They described average radiological union time as 14 - 25 weeks, and 13-20 weeks respectively.

In our study, out of 58 patients, the range of motion (ROM) of the knee joint at final follow-up (2 years) was 110 degrees and more in 28 (48.3\%) patients with good to excellent functional outcome. In 13 (22.4\%) patients, we succeed in achieving 91-109 degrees ROM with satisfactory functional outcome. Although, we failed to obtain a satisfactory ROM in 17 (29.3\%) patient up-to their final follow-up. Some of these patients underwent knee mobilization. They refused for any additional surgery to increase ROM and continued with non-operative care. The average range of motion of the knee joint was 105.5 degrees in our study. The average range of motion of the knee joint was 110 degrees in the study of Markmiller et al. [24].

In this study, the results expressed according to the Schatzker \& Lambert's criteria [13]. In this study, out of total 58 cases, results were as excellent in 28 (48.3\%) cases, good in 11 (19\%) cases, fair in $13(22.4 \%)$ cases, and failure in 6 (10.3\%) cases. Paknikar KP et al. [25] reported their study result as excellent in $32 \%$ patients, good in 28\%, fair in 34\%, and poor in $6 \%$ patients. Padha $\mathrm{K}$ et al. [26], described their study results as excellent in $44 \%$, good in $32 \%$, fair in $16 \%$, and failure in $8 \%$ patients.

In the present study, out of 58 cases, three (5.2\%) patients had superficial surgical site infections. These cases successfully treated with proper dressings and oral antibiotics. Although, there was no long term adverse effect on fracture healing or rehabilitation of these patients due to this superficial infection. We observed two cases (3.4\%) withdeep surgical site infections. Both cases successfully managed with debridement, adequate lavage, and intravenous antibiotics. Kregor et al. [27] reported in their studythat deep infection manifested in 3\% of their patients.

Knee stiffness observed in 9 (15.5\%) patients. It was the most common complication of our study. We encountered $3(5.2 \%)$ cases with mild limb length discrepancy or limb shortening $<2 \mathrm{~cm}$. This mild shortening was well compensated by equinus position at ankle joint, and was acceptable to the patients. We observed a total $3(5.2 \%)$ patients with implant failure within the first 12 weeks of primary surgery. Out of 58 patients, we noticed 4 (6.9\%) patients with non-union at fracture site at their one-year follow-ups. All these cases underwent revision surgery. The procedure carried out was- implant removal and re-fixation with longer DF-LCP with autologous bone grafting from the ipsilateral iliac crest, and satisfactory functional outcomes achieved after the revision surgery. Out of 58 patients, we noted secondary arthritis in 5 (8.6\%) patients, for which some of these patients have to go replacement arthroplasty at a later stage. All these complications were comparable with the complications mentioned in the previous studies [28, 29, 30].

\section{a) Limitations of the study}

One of the main limitation of our study was the small sample size. The small sample size influences the evaluation of outcomes, as it can overrate the results. Furthermore, the study includes the single method of fracture fixation with distal femoral locking compression plate (DF-LCP) only. At the same time, other various fixation methods could have also been used for comparison and to conclude more significant results.

\section{Conclusion}

Distal femoral locking compression plate (DFLCP) is an extra-medullary load-bearing device, which is an ideal implant to prevent metaphyseal collapse, malrotation and to maintain limb length especially in osteoporotic and severely comminuted distal femur fractures with intra-articular extension. DF-LCP has combi holes in the stem and locking bolts in the expanded head area. With the proper patient selection, it holds the metaphyseal bone firmly in highly unstable distal femur fractures, and simultaneously, it provides stable fixation in the distal femoral shaft to promote callus formation and allows early mobilization and early weight-bearing with acceptable complication rates. 


\section{Acknowledgement: None}

\section{Declarations:}

Funding: No funding sources.

Conflict of interest: None declared.

Ethical approval: This study was approved by the institutional ethics committee.

\section{References Références Referencias}

1. Court-Brown CM, Caesar B. Epidemiology of adult fractures: a review. Injury. 2006; 37:691-697.

2. Martinet $\mathrm{O}$, Cordey J, Harder $\mathrm{Y}$, Maier A, Buhler M, Barraud GE. The epidemiology of fractures of the distal femur. Injury. 2000; 31(3):C62-C63.

3. Schatzker J, Home G, Waddell J (1974) The Toronto experience with the supracondylar fracture of the femur 1966-1972. Injury 6: 113-123.

4. Hoffmann MF, Jones CB, Sietsema DL, Tornetta P, Koenig SJ. Clinical outcomes of locked plating of distal femoral fractures in a retrospective cohort. Journal of orthopaedic surgery and research. 2013 Nov 27; 8(1):43.

5. Whittle AP, Wood II GW. Campbell's operative orthopaedics, Chapter 51, In: Fractures of lower extremity, 10th ed, Mosby Inc 2003, 3.

6. Palcide RJ, Lonner JH. Fractures of the distal femur. Current opinion in orthopaedics 1999; 10(1):2-9.

7. Stewart MJ, Sisk TD, Wallace SL. Fractures of the distal third of femur. J Bone Joint Surg. 1966; 48A:784-807.

8. Neer CS, Grantham SA, Shelton ML. Supracondylar fracture of the adult femur. J Bone Joint Surg 1967; 49A:591-613.

9. Firoozbakhsh K, Behzadi K, Decoster TA, Moneim MS, Naraghi FF. Mechanics of retrograde nail versus plate fixation for supracondylar femur fractures. J Orthop Trauma. 1995; 9:152-7.

10. Thomas TL, Meggit BF. A comparative study of methods for treating fractures of the distal femur. J Bone Joint Surg. 1981; 63B(1):3-6.

11. Siliski JM, Mahring M, Hofer HP. Supracondyalarintercondylar fracture of the femur. Bone Joint Surg. 1989; 71:95-104.

12. Rockwood and Green's fractures in adults/ 8th edition/vol 2/section four lower extremity/chap 53/page no 2229-2268.

13. Schatzker J, Lambert DC. Supracondylar Fractures of the Femur. Clin Orthop 1979; 138:77-83.

14. Wagner M, Frenk A, Frigg R. New concepts for bone fracture treatment and the Locking Compression Plate. Surg Technollnt, 2004; 12:271-7.

15. Gustilo RB, Anderson JT. Prevention of infection in the treatment of one thousand and twenty-five open fractures of long bones: Retrospective and prospective analyses. J Bone Joint Surg Am. 1976; 58:453-8.
16. Mahesh DV, Gunnaiah V. Management of Distal Femur Fracture by Locking Compression Plate. International Journal of Health Sciences and Research (IJHSR). 2014; 4(5):235-40.

17. Rao LL, Kumar TD, Paleti ST, et al. Evaluation of functional outcome after open reduction and internal fixation of distal femur fractures by locking compression plate. J. Evid. Based Med. Healthc. 2016; 3(73), 3966-3972.

18. Kubiak EN, Fulkerson E, Strauss E, Egol KA: The evolution of locked plates. J Bone Joint Surg Am 2006, 88 (Suppl 4):189-200.

19. Wagner M. General principles of clinical use of LCP. Injury 2003; 34:5-B31-42

20. Siliski JM, Mahring M, Hofer P. Supracondylarintercondylar fractures of the femur treated by internal fixation. J Bone Joint Surg. 1989; 71:95-104.

21. Yeap EJ, Deepak AS. Distal femoral locking plate fixation in distal femoral fractures. Malaysian Orthopaedic J 2007; 1:12-17

22. Rajaiah D, Ramana $Y$, Srinivas $K$ et al. A study of surgical management of distal femoral fractures by distal femoral locking compression plate osteosynthesis. J Evid. Based Med. Healthc. 2016; 3(66): 3584-3587.

23. Kim KJ, Lee SK, Choy WS et al. Surgical treatment of $\mathrm{AO}$ type $\mathrm{C}$ distal femoral fractures using locking compression plate (LCP-DF Synthes). J Korean Fract Soc. 2010; 23(1):20-25.

24. Markmiller M, Konard G, Sudkamp N. femur- LISS and Distal Femoral Nail for fixation of distal femoral fractures. Clin Orthop 2004; 426:252-57

25. Kiran P. Paknikar, Shekhar Malve, G. S. Kulkarni. Supracondylar fracture of femur: our experience of treatment with locking compression plate from rural Maharashtra. Int J Res Orthop. 2019 Mar; 5(2):232-236.

26. Kanav Padha, Sandeep Singh, Abdul Ghani, Harish Dang. Distal Femur Fractures and its Treatment with Distal Femur Locking Plate. JK Science. 2016:18(2):76-80.

27. Kregor PJ, Stannard J, Zlowodski M, et al. Treatment of distal femoral fractures using Less Invasive Stabilization system. Surgical technique and early clinical results in 103 fractures. J Orthop Trauma 2004: 18(8): 509-20

28. Schutz M, Muller M, Regazzoni $P$, et al. Use of less invasive stabilization system in patients with distal femoral fractures: a prospective multicentric study. Acta OrthopTrauma Surgery 2005; 125(2) 102-08

29. Bolhofner BR, Carmen B, Clifford $P$. The results of open reduction and internal fixation of distal femur fractures using a biologic (indirect) reduction technique. J Orthop Trauma. 1996; 10:372-7.

30. Ostrum RF, Geel C. Indirect reduction and internal fixation of supracondylar femur fractures without bone graft. J OrthopTrauma. 1995; 9:278-84. 